\title{
Closed form solution of plane-parallel turbulent flow along an unbounded plane surface
}

\author{
Bohua Sun $^{1}$ \\ ${ }^{1}$ School of Civil Engineering 83 Institute of Mechanics and Technology \\ Xi'an University of Architecture and Technology, Xi'an 710055, China \\ email: sunbohua@xauat.edu.cn
}

(Dated: 28 Sept. 2021)

\begin{abstract}
In this letter, a century-old problem is studied; namely, to find a unified analytic description of the non-uniform distribution of mean velocity across the entire domain of turbulent flow for all Reynolds numbers within the framework of the Prandtl mixing length theory. Considering the Prandtl mixing length model, a closed form solution of the mean velocity profile of plane turbulent flow is obtained. The profiles of several useful quantities are given, such as turbulent viscosity, Reynolds turbulent stress, Kolmogorov's scaling law, and energy dissipation density. It is shown that the energy dissipation density at the surface is finite, whereas Landau's energy dissipation density is infinite. The closed form solution reveals that the universality of the turbulent velocity logarithmic profile no longer holds, but the von Kármán constant is still universal. Furthermore, a new formulation of the resistance coefficient of turbulent flow in pipes is given in implicit form.
\end{abstract}

Keywords: turbulent flow, plane, Prandtl mixing length, Reynolds number, boundary layer

\section{INTRODUCTION}

The theory of the turbulence boundary layer dates to the beginning of the 20th century. In 1904, Prandtl [1] proposed that the high Reynolds flow of parallel walls produces a very thin boundary layer at the wall that exhibits basically non-viscous motion; however, the viscous effect within the boundary layer cannot be ignored. In 1908, Blasius [2] obtained a complete description of the laminar boundary layer, which resulted in the classical laminar boundary-layer theory. However, almost all boundary-layer movements in nature are turbulent rather than laminar [3-9], meaning related problems involve predicting the turbulent kinetic energy profile in addition to the mean velocity profile, which have been investigated extensively by [10-15] from perspectives of the Lie symmetry.

\section{A PLANE-PARALLEL TURBULENT FLOW ALONG AN UNBOUNDED SMOOTH PLANE SURFACE}

We consider here a plane-parallel turbulent flow along an unbounded smooth plane surface (wall), and take the direction of the flow as the $x$ axis and the plane of the surface as the $x z$ plane, so $y$ is the direction orthogonal to the surface. Assuming that the turbulent flow is steady with no pressure gradient along the $x$ axis, the $y$ and $z$ components of the mean velocity are zero, and all of the quantities depend only on $y$.

The Reynolds-averaged Navier-Stokes equation of the plane-parallel turbulence is reduced to $\mu \frac{d^{2} \bar{u}}{d y^{2}}+\frac{d \tau_{x y}^{\prime}}{d y}=0$, where $\mu$ is the dynamical viscosity, $\bar{u}$ is the mean velocity, and $\tau_{x y}^{\prime}=-\rho \overline{u^{\prime} v^{\prime}}$ is the Reynolds stress. Under boundary conditions, i.e., $y=0: \bar{u}=0, u^{\prime}=0, v^{\prime}=0$ and $\mu \frac{d \bar{u}}{d y}=\tau_{w}$, the above equation can be integrated to

$$
\mu \frac{d \bar{u}}{d y}-\rho \overline{u^{\prime} v^{\prime}}=\tau_{w},
$$

where $\tau_{w}$ is the wall friction force on a unit area of the surface. This force is clearly in the $x$ direction. The quantity $\tau_{w}$ is the constant flux of the $x$ component of momentum transmitted by the fluid to the surface per unit time. The first term on the left-hand side of Eq. 1 represents the effect of viscosity on the mean flow, whereas the second term is the Reynolds stress. In turbulent flow located some distance away from a wall, the Reynolds stress is of considerably greater magnitude than the viscous stress; however, the role of viscous stress increases as the distance to a smooth wall decreases until finally, at the wall, viscosity predominates.

\section{THE PRANDTL MIXING LENGTH THEORY OF THE PLANE-PARALLEL TURBULENT FLOW AND PRANDTL'S ASYMPTOTIC SOLUTIONS}

According to the Prandtl mixing length theory [4], the Reynolds stress is proposed to be $\tau_{x y}^{\prime}=\rho \ell^{2}\left|\frac{d \bar{u}}{d y}\right| \frac{d \bar{u}}{d y}$, where the mass density is $\rho$, the mixing length is constructed by dimensional arguments as $\ell=\kappa y$, and $\kappa$ is a numerical constant, namely, the von Kármán constant. Hence, Eq. 1 becomes $\mu \frac{d \bar{u}}{d y}+\rho(\kappa y)^{2}\left|\frac{d \bar{u}}{d y}\right| \frac{d \bar{u}}{d y}=\tau_{w}$, namely,

$$
\begin{aligned}
& \frac{d \bar{u}}{d y}<0: \quad \mu \frac{d \bar{u}}{d y}-\rho(\kappa y)^{2}\left(\frac{d \bar{u}}{d y}\right)^{2}=\tau_{w}, \\
& \frac{d \bar{u}}{d y}>0: \quad \mu \frac{d \bar{u}}{d y}+\rho(\kappa y)^{2}\left(\frac{d \bar{u}}{d y}\right)^{2}=\tau_{w},
\end{aligned}
$$

and the boundary condition is

$$
y=0: \bar{u}=0 .
$$


So far, no complete solutions have been obtained for either Eq. 2 or Eq. 3. Instead, asymptotic solutions have been constructed in two different regions (or sub-layers) [7].

In the inertial sub-layer, the first terms of Eq. 2 and Eq. 3 are neglected, leading to $\rho(\kappa y)^{2}\left(\frac{d \bar{u}}{d y}\right)^{2}=\tau_{w}$, the solution of which is a well-known Prandtl logarithmic law,

$$
\frac{\bar{u}}{u_{\tau}}=\frac{1}{\kappa} \log \left(\frac{y \bar{u}}{\nu}\right)+\alpha-\frac{1}{\kappa} \log \alpha,
$$

where $u_{\tau}=\sqrt{\tau_{w} / \rho}$ and $\nu=\mu / \rho$.

According to Nikuradse's famous experiments [6], data fitting gives $\kappa=0.4$ and $\alpha=11.5$; hence, the Prandtl log-law is

$$
\frac{\bar{u}}{u_{\tau}}=5.75 \log \left(\frac{y \bar{u}}{\nu}\right)+5.5
$$

This expression becomes infinite at the boundary $y=0$ and is inapplicable at very small distances $y$ from the surface, since the effect of viscosity near the surface becomes non-negligible [8].

To fix the singularity problem, traditionally a viscous sub-layer is introduced in which the viscosity of the fluid begins to be important. The second terms of Eqs. 2 and 3 can be neglected, leading to $\mu \frac{d \bar{u}}{d y}=\tau_{w}$, the solution of which is the Prandtl linear law: $\bar{u}=\tau_{w} y / \mu$.

After obtaining these two sub-layer solutions, matching them into one segmental solution is done as follows:

$$
u^{+}=\eta+(5.394-\eta+2.5 \ln \eta) H(\eta-5.394),
$$

where $H(\ldots)$ is the Heaviside step function, $u^{+}=\frac{\bar{u}}{u_{\tau}}$, and $\eta=\frac{y u_{\tau}}{\nu}$ (note: $\eta$ is denoted as $y^{+}$in some publications).

\section{A CENTURY-OLD MATHEMATICAL PROBLEM IN TURBULENCE}

Mathematically speaking, the above two segmental solutions, including linear and log-law solutions, are incomplete, because they are not whole domain solutions. Rather, it is a local solution of the matching area between the inertial sub-layer and viscous sub-layer of turbulence. Between the inertial region and viscous sub-layer, there is an intermediate region whose empirical solution has not been obtained [7, 8, 10-15, 17, 18].

Finding a unified analytic description of the nonuniform distribution of mean velocity and turbulence intensities across the entire domain of turbulent flow for all Reynolds numbers is an open problem dating back to Prandtl's pioneering work in 1904 [9-15, 17, 18].

\section{CLOSED FORM SOLUTION}

To find a singularity-free and consistent solution valid in the whole domain of $y$, we return to the complete governing equations, Eqs. 2 and 3, and work to find their solutions without any approximations, as in the preceding section. We find the exact solutions for both Eqs. 2 and 3, but the solution of Eq. 2 is not physically possible since two terms of the solution are infinite at $y=0$. The only physically possible equation is Eq. 3, which can be rewritten as

$$
\frac{d u^{+}}{d \eta}+\kappa^{2} \eta^{2}\left(\frac{d u^{+}}{d \eta}\right)^{2}=1
$$

together with the boundary condition

$$
\eta=0: u^{+}=0
$$

Equation 8 has two solutions, one a singular solution, $u^{+}=-\int \frac{1+\sqrt{1+4 \kappa^{2} \eta^{2}}}{2 \kappa^{2} \eta^{2}} d \eta=\frac{1+\left(1+4 \kappa^{2} \eta^{2}\right)^{3 / 2}}{2 \kappa^{2} \eta}-$ $2 \eta \sqrt{1+4 \kappa^{2} \eta^{2}}-\frac{1}{\kappa} \ln \left(2 \kappa \eta+\sqrt{1+4 \kappa^{2} \eta^{2}}\right)+C_{0}$ due to the singularity of $\frac{1+\left(1+4 \kappa^{2} \eta^{2}\right)^{3 / 2}}{2 \kappa^{2} \eta}$ at $\eta \rightarrow 0$; and the other a singularity-free solution, $u^{+}=\int \frac{-1+\sqrt{1+4 \kappa^{2} \eta^{2}}}{2 \kappa^{2} \eta^{2}} d \eta=$ $\frac{1}{2 \kappa^{2} \eta}+\int \frac{\sqrt{1+4 \kappa^{2} \eta^{2}}}{2 \kappa^{2} \eta^{2}} d \eta$

Introducing a transformation $2 \kappa \eta=\sinh \xi$, hence $\sqrt{1+4 \kappa^{2} \eta^{2}}=\cosh \xi$ and $d \eta=\frac{\cosh \xi}{2 \kappa} d x$, the integration becomes $\int \frac{\sqrt{1+4 \kappa^{2} \eta^{2}}}{2 \kappa^{2} \eta^{2}} d \eta=\frac{1}{\kappa} \int\left(\frac{\cosh \xi}{\sinh \xi}\right)^{2} d \xi=\frac{1}{\kappa}(\xi-\operatorname{coth} \xi)$. Notice $\xi=\ln \left(2 \kappa \eta+\sqrt{1+4 \kappa^{2} \eta^{2}}\right)$ and $\operatorname{coth} \xi=\frac{\sqrt{1+4 \kappa^{2} \eta^{2}}}{2 \kappa \eta}$. Therefore, the closed form solution of Eq. 8 is obtained as

$$
u^{+}=\frac{1}{\kappa} \ln \left(2 \kappa \eta+\sqrt{1+4 \kappa^{2} \eta^{2}}\right)-\frac{2 \eta}{1+\sqrt{1+4 \kappa^{2} \eta^{2}}}+C
$$

where $C_{0}$ and $C$ are integration constants that can be determined by the boundary condition in Eq. 9 .

The solution in Eq.10 is singularity free because of the cancellation of singularity of the term $\lim _{\eta \rightarrow 0}\left(\frac{2 \eta}{1+\sqrt{1+4 \kappa^{2} \eta^{2}}}\right)=0$, which leads to the limit $\lim _{\eta \rightarrow 0} u^{+}=C$. Applying the boundary condition in Eq. 9 gives $C=0$. Hence, the closed form solution of Eq. 8 is given by

$$
u^{+}=\frac{1}{\kappa} \ln \left(2 \kappa \eta+\sqrt{1+4 \kappa^{2} \eta^{2}}\right)-\frac{2 \eta}{1+\sqrt{1+4 \kappa^{2} \eta^{2}}} .
$$

Strictly speaking, the solution in Eq. 11 reveals that the velocity profile of plane-turbulent flow is not logarithmic as stated in [17], owing to the exist of the 2nd term " $-\frac{2 \eta}{1+\sqrt{1+4 \kappa^{2} \eta^{2}}}$ ". However, the logarithmic law of the turbulent velocity profile can still be approximately 
maintained for very large $\eta$. Near the surface, the velocity profile is not linear.

This singularity-free solution is valid for the whole domain of $\eta \in[0, \infty]$. The only unknown in the solution is the von Kármán constant $\kappa$, which could be determined by data fitting from the experiments [6] to be $\kappa \approx 0.4$, confirming that the von Kármán constant is still a universal constant.

Due to the identity of hyperbolic arcsine $\sinh ^{-1} x=$ $\ln \left(x+\sqrt{1+x^{2}}\right), x \in(-\infty, \infty)$, the closed form solution can also be expressed as follows

$$
u^{+}=\frac{1}{\kappa} \sinh ^{-1}(2 \kappa \eta)-\frac{2 \eta}{1+\sqrt{1+4 \kappa^{2} \eta^{2}}} .
$$

\section{THE CLOSED FORM SOLUTION VS. THE PRANDTL LOG-LAW SOLUTION}

To visualize the difference between the segmental solution and the closed form solution, Eqs. 7 and 11 are depicted in Fig. 1.

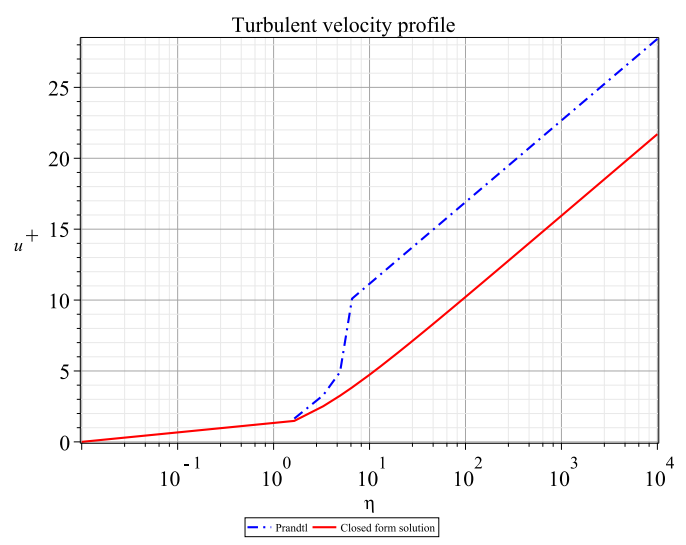

FIG. 1: The $u^{+}-\log \eta$ curve. Eq. 7 is represented by the blue dashline, and Eq. 11 is represented by the red line. The plot indicates that the mean velocity of the Prandtl solution is larger than the velocity obtained by the closed form solution as a result of neglecting the viscosity in Prandtl's solution.

\section{THE CLOSED FORM SOLUTION VS. DIRECT NUMERICAL SOLUTION (DNS)}

For validation, a comparison between the closed form solution in Eq. 11 and direct numerical simulation (DNS) solution $[18,19]$ is depicted in Fig. 2

Although we successfully obtained the closed form solution of plane turbulent flow based on the Prandtl mixing length model $\ell^{+}=\kappa \eta$, where $\ell^{+}=\ell u_{\tau} / \nu$, unfortunately, Fig. 2 shows that the closed form solution does not perfectly agree with the DNS solution [18, 19] for a smooth wall. This disagreement is caused by the Prandtl

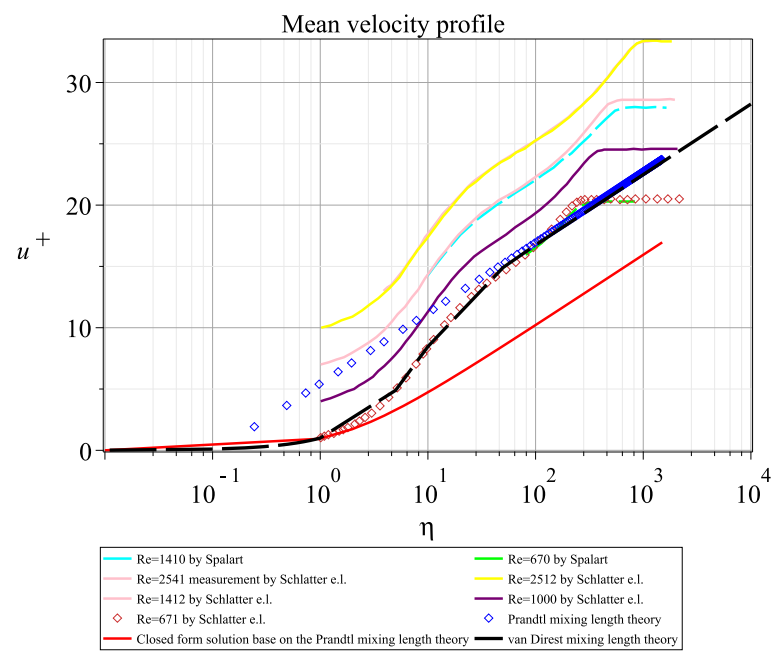

FIG. 2: Comparisons: the closed form solution, the Prandtl solution, van Driest mixing length theory [21], the direct numerical simulation (DNS) solution [18, 19].

mixing length theory and nothing to do with the mathematical rigorous treatment of the Eq.(e-4) and its closed form solution. Therefore, if we consider the DNS solution as a high-performance solution, to fix the mess we must modify the Prandtl mixing length model to make the solution as close to the DNS solution as possible.

\section{VAN DRIEST'S MODIFICATION OF THE PRANDTL MIXING LENGTH THEORY}

Regarding the modification, we consider an infinite flat plate undergoing simple harmonic oscillation parallel to the plate in an infinite fluid. According to Stokes [20], the amplitude of the motion diminishes with increasing distance from the surface (wall) as a consequence of the factor $\exp (-y / A)$, where $A$ is a constant depending the frequency of oscillation of the plate and kinematic viscosity $\nu$ of the fluid. Hence, van Driest [21] pointed out that when the plate is fixed and the fluid oscillates relative to the plate, the factor $[1-\exp (-y / A)]$ must be applied to the fluid oscillation to obtain the damping effect on the wall. Furthermore, van Driest believed that fully developed turbulent motion occurs only beyond a distance sufficiently remote from the wall, and eddies are not damped by the nearness of the wall. Indeed, near a wall, the damping factor is $[1-\exp (-y / A)]$ for each mean velocity fluctuation, and the Reynolds stress should be modified to become

$$
\begin{aligned}
\tau_{x y}^{\prime} & =\kappa[1-\exp (y / A)](d \bar{u} / d y)^{2} \\
& =\kappa\left[1-\exp \left(\eta / A^{+}\right)\right]\left(d u^{+} / d \eta\right)^{2},
\end{aligned}
$$

in order to take into account the mean motion all the way to a smooth wall, where $\kappa \simeq 0.4, A^{+} \simeq 26$. 
With the van Driest mixing length theory, Eq. 8 should be modified to the following:

$$
\frac{d u^{+}}{d \eta}+\kappa^{2} \eta^{2}\left[1-\exp \left(-\eta / A^{+}\right)\right]^{2}\left(\frac{d u^{+}}{d \eta}\right)^{2}=1
$$

whose solution can be expressed in an integration [21]

$$
u^{+}=\int \frac{2}{1+\sqrt{1+4 \kappa^{2} \eta^{2}\left[1-\exp \left(-\eta / A^{+}\right)\right]^{2}}} d \eta,
$$

which unfortunately has no analytical solution. However, we can solve it numerically. The solution of Eq. 15 is depicted in Fig. 2. Fig. 2 shows that the van Driest model agrees well with the DNS solution.

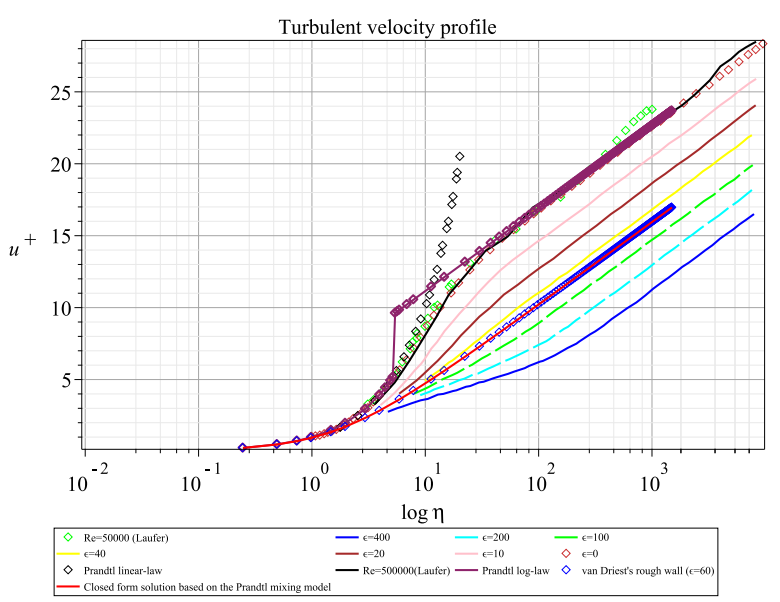

FIG. 3: Comparison between the closed form solution, van Driest's solution [21], and Laufer's experiments [22]. The dimensionless roughness is $\epsilon=u_{\tau} \lambda / \nu$, and $\lambda$ is the roughness height.

We also depict the closed form solution in the Fig. 3. It is surprising to see that the closed form solution perfectly matches the van Driest solution for a rough wall $(\epsilon=60)$, which indicates that the Prandtl mixing length theory might be more suitable for rough walls than smooth walls.

The reason of behind this scenario is because the van Driest mixing length theory applies to a smooth wall under the condition that the proximity to the wall has a stabilizing effect upon eddies owing to viscosity. The stabilizing effect of the wall can, however, be nullified by introducing an artificial mixer at the wall-viz., roughness. When sufficient roughness is introduced to stir up motion near the wall (vortex generation), the factor $[1-\exp (-y / A)]$ should disappear from the above formulations, in which case the van Driest mixing length theory becomes equivalent to the Prandtl mixing length model [21]. This is why the Prandtl mixing length theory is suitable for rough walls and the van Driest mixing length theory is suitable for smooth walls [21]. This argument is well agree with van Driest's pespective [21].

This situation is very much like Koiter's post-buckling theory of structures with geometrical imperfections, in which Koiter postulates there are no perfect structures, and geometrical imperfection is the key factor affecting the buckling behaviors of structures. To better predict buckling loads, imperfections must be taken into account, so Koiter developed a post-buckling theory that gives much more reliable buckling loads for structures with geometrical imperfections [27-32]. In the same way, there are no perfectly smooth walls, meaning a real wall will always have some kind of roughness. Therefore, the closed form solution based on the Prandtl mixing length theory will give a better prediction of the turbulent flow.

\section{SOME QUALITIES CALCULATED FROM THE CLOSED FORM SOLUTION}

With the obtained closed form solution in Eq. 11, we can calculate some useful quantities, as follows:

(1) The turbulent viscosity is $\nu_{T}=(\kappa y)^{2} \frac{d \bar{u}}{d y}=$ $\nu \kappa^{2} \eta^{2} \frac{d u^{+}}{d \eta}$, where $\frac{d u^{+}}{d \eta}=\frac{-1+\sqrt{1+4 \kappa^{2} \eta^{2}}}{2 \kappa^{2} \eta^{2}}=\frac{2}{1+\sqrt{1+4 \kappa^{2} \eta^{2}}}$; hence,

$$
\nu_{T}=\frac{1}{2} \nu\left(-1+\sqrt{1+4 \kappa^{2} \eta^{2}}\right) .
$$

This relation reveals that the turbulent viscosity is not constant but rather increases with $\eta$. It is depicted in Fig. 4.

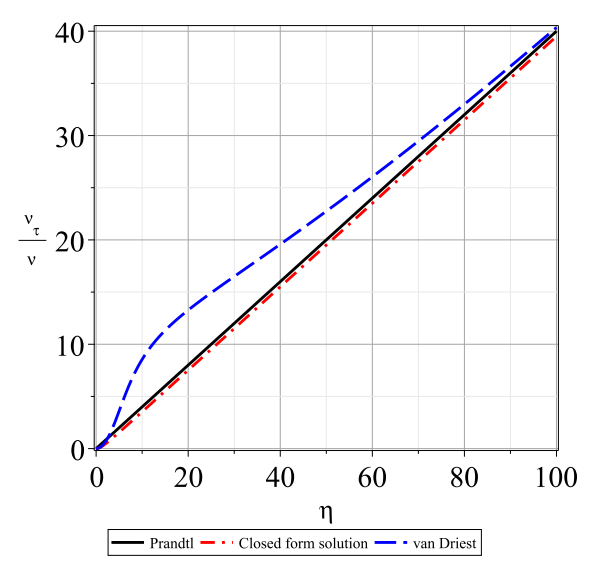

FIG. 4: The turbulent viscosity ratio, the van Driest turbulent viscosity [21] is larger than both the closed form solution's and Prandtl's

(2) The Reynolds stress is given by

$$
\tau_{x y}^{\prime}=\rho u_{\tau}^{2} \kappa^{2} \eta^{2}\left(\frac{d u^{+}}{d \eta}\right)^{2}=\frac{4 \rho u_{\tau}^{2} \kappa^{2} \eta^{2}}{\left(1+\sqrt{1+4 \kappa^{2} \eta^{2}}\right)^{2}} .
$$

The Reynolds stress ratio $\frac{\tau_{x y}^{\prime}}{\rho u_{\tau}^{2}}$ of the segmental solution and the closed form solution is depicted in Fig. 5 . 


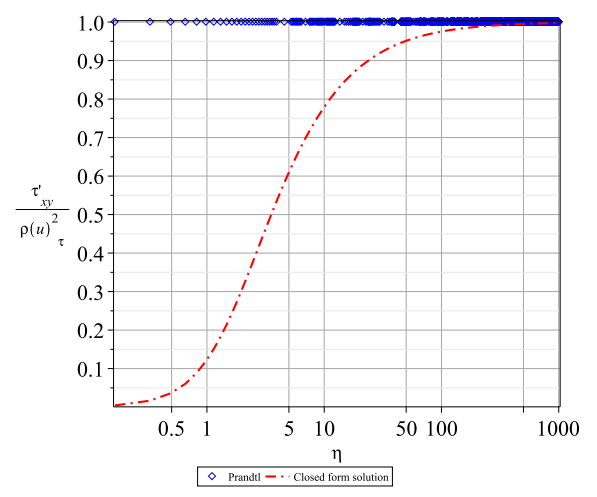

FIG. 5: The Reynolds stress ratio $\frac{\tau_{x y}^{\prime}}{\rho u_{\tau}^{2}}$, generally speaking, the Reynolds stress calculated from the closed form solution increases with the distance from the surface before it becomes a constant, but the segmental solution gives constant Reynolds stress $\frac{\tau_{x y}^{\prime}}{\rho u_{\tau}^{2}}=1$ for all $\eta$.

(3) According to Landau [8], the mean energy flux density is $\langle q\rangle=\bar{u} \tau_{w}$, and the energy dissipation density in the turbulent flow is

$$
\begin{aligned}
\varepsilon & =\frac{1}{\rho} \frac{d<q>}{d y}=\frac{1}{\nu}\left(\frac{\tau_{w}}{\rho}\right)^{2} \frac{d u^{+}}{d \eta}=\frac{u_{\tau}^{4}}{\nu} \frac{d u^{+}}{d \eta} \\
& =\frac{u_{\tau}^{4}}{\nu} \frac{2}{1+\sqrt{1+4 \kappa^{2} \eta^{2}}},
\end{aligned}
$$

which gives $\lim _{\eta \rightarrow 0} \varepsilon=\frac{u_{\tau}^{4}}{\nu}$, i.e., a power law $u_{\tau}^{4}$; in contrast, Landau's energy dissipation density [8], $\varepsilon_{\text {Landau }}=$ $\left(\frac{\tau_{w}}{\rho}\right)^{3 / 2} \frac{1}{\kappa y}=\frac{u_{\tau}^{4}}{\nu} \frac{1}{\kappa \eta}$, is infinite at the surface $\eta=0$, indicating that maintaining turbulent flow requires supplying an infinite energy source, which is physically impossible. Our energy dissipation density and Landau's are depicted in Fig. 6.

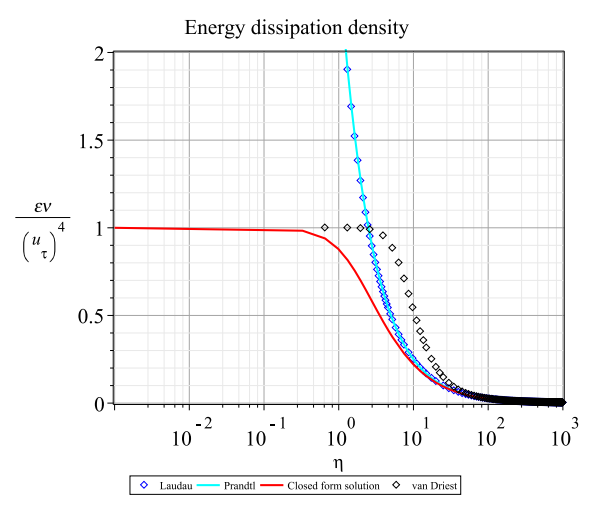

FIG. 6: The energy dissipation density ratio $\frac{\varepsilon \nu}{u_{\tau}^{4}}$ decreases to null rapidly as $\eta$ increases away from the surface.

(4) According to Kolmogorov [23-26], we have $E(k)=$
$1.5 \varepsilon^{2 / 3} k^{-5 / 3}$, namely,

$$
E(k)=1.5 \nu^{-2 / 3} k^{-5 / 3} u_{\tau}^{8 / 3}\left(\frac{2}{1+\sqrt{1+4 \kappa^{2} \eta^{2}}}\right)^{2 / 3}
$$

where $E(k)$ is the kinetic energy per unit mass of fluid in eddies with wave number $k$. Hence, at the surface $\eta=0$, we have $E(k)_{\eta=0}=1.5 u_{\tau}^{8 / 3} k^{-5 / 3} \nu^{-2 / 3}$, which is depicted in Fig. 7.

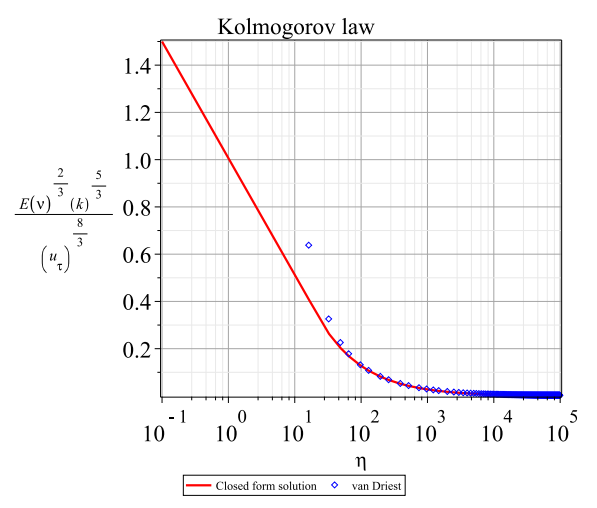

FIG. 7: Kolmogorov's law: Eq. 11 (in red) and van Driest [21] (in blue).

Figure 7 shows that the turbulent energy dissipation is mainly concentrated in a narrow region close to the boundary surface and decays rapidly away from the surface.

\section{APPLICATION TO THE FRICTION OF CIRCULAR PIPES}

As an application of the solution in Eq. 11, let us now apply Eq. 11 to turbulent flow in a pipe with length $l$ and radius $a$. The area near the walls of the pipe may be approximately regarded as a plane, and the velocity profile must be given by Eq. 11. The mean velocity $\bar{u}$ of the flow in the pipe is calculated by $\bar{u}=Q /\left(\rho \pi a^{2}\right)$, where $Q$ is the volume of fluid that passes through a crosssection of the pipe per unit time divided by the crosssection area. Since the frictional force per unit area of the wall is $\tau_{w}=\rho u_{\tau}^{2}$, the total frictional force is $2 \pi a l \rho u_{\tau}^{2}$. Assuming the pressure gradient $\Delta p / l$ maintains the flow, the force on the cross-section is $\pi a^{2} \Delta p$. Equating the two forces, namely $\pi a^{2} \Delta p=2 \pi a l \rho u_{\tau}^{2}$, leads to flow pressure gradient $\Delta p / l=2 \rho u_{\tau}^{2} / a$.

We must point out that the obtained closed form solution of Eq. 11 is only valid for turbulent flow with no pressure gradient, namely, $\Delta p / l=2 \rho u_{\tau}^{2} / a=0$. Strictly speaking, the closed form solution cannot be used to deal with flow with pipe friction and a flow pressure gradient. 
We can still use the solution as an approximation if we assume the pressure gradient is infinitesimal, for instance, in the case of large radius $a$, i.e., a large Reynolds number. Therefore, van Driest argues that the above results for flow in the vicinity of smooth walls are also approximately valid with a pressure gradient in the direction of flow because the shear stress near the wall is approximately equal to the wall stress [21]. In the following, all formulations are derived under the condition of small $\Delta p / l$.

The dependence of the resistance coefficient $\lambda=$ $\frac{2 a \Delta p / l}{(1 / 2) \rho \bar{u}^{2}}$ on the dimensionless Reynolds number $R e=$ $2 a \bar{u} / \nu$ is given in implicit form by the equation

$$
\begin{aligned}
\frac{1}{\sqrt{\lambda}} & =\frac{1}{\kappa^{2} \operatorname{Re} \sqrt{\lambda}}-\frac{\ln 2}{\sqrt{2} \kappa}+\frac{\sqrt{16+2 \kappa^{2} R e^{2} \lambda}}{4 \kappa^{2} \operatorname{Re} \sqrt{\lambda}} \\
& +\frac{\sqrt{2}}{4 \kappa} \ln \left(\sqrt{2} \kappa \operatorname{Re} \sqrt{\lambda}+\sqrt{16+2 \kappa^{2} R e^{2} \lambda}\right),
\end{aligned}
$$

where $a$ is the pipe radius, and $\Delta p / l$ is the pressure gradient. If we set $\kappa=0.4$, we have

$$
\begin{aligned}
\frac{1}{\sqrt{\lambda}} & =\frac{6.25}{\operatorname{Re} \sqrt{\lambda}}-1.225-1.5625 \frac{\sqrt{16+0.32 R e^{2} \lambda}}{\operatorname{Re} \sqrt{\lambda}} \\
& +0.8839 \ln \left(0.5657 \operatorname{Re} \sqrt{\lambda}+\sqrt{16+0.32 \operatorname{Re}^{2} \lambda}\right) .
\end{aligned}
$$

Eq. 21 and the Prandtl $\log$-law, $1 / \sqrt{\lambda}=2 \log (\operatorname{Re} \sqrt{\lambda})-$ 0.8, are depicted in Fig. 8.

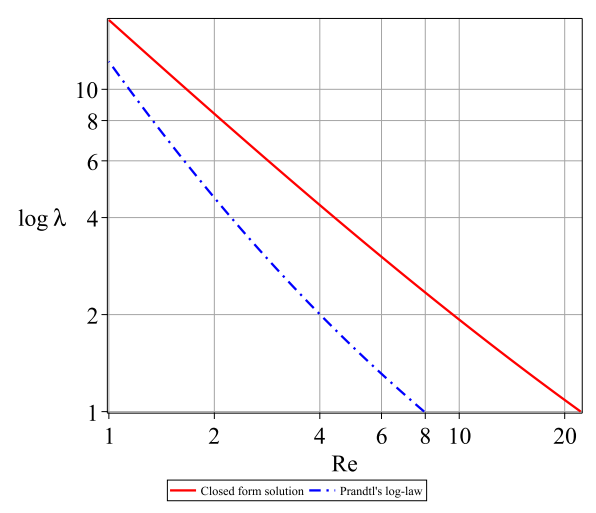

FIG. 8: Pipe resistance coefficient. The Prandtl log-law is represented by a dotted line, and Eq. 21 is represented by a solid line. The resistance coefficient derived from Eq. 21 is higher than that derived from the Prandtl log-law since the closed form solution includes the viscous influence, whereas the Prandtl log-law does not.

\section{CONCLUSIONS}

To the best of the author's knowledge, the solution in Eq. 11 is the first closed form solution of plane turbulent flow ever obtained in the context of rigorous mathematics. This study may help to have a better understanding of turbulence phenomena.

\section{Acknowledgment}

The author wishes to express his appreciation to his student, Mr. Zhe Liu, for preparing Figs. 2 and 3.

\section{Availability of data}

The data supporting the findings of this study are available from the corresponding author upon reasonable request.

[1] L. Prandtl, On fluid motions with very small friction (in german). Third International Mathematical Congress, Heidelberg (1904)

[2] H. Blasius, Grenzschichten in Fljssigkeiten mit kleiner Reibung. Z. Math. Phys., 56 1-37(1908).

[3] Th. Von Kámán, über leminere und turbulence Reibung. (On Laminer and Turbulent Friction.) Z.A.M.M. 1 (1921).

[4] L. Prandtl, Bericht über Untersuchungen zur ausgebildeten Turbulenz, Z. Angew. Math. Mech. 5, 136 (1925).

[5] C. M. Millikan, A critical discussion of turbulent flows in channels and circular tubes, in Proceedings of the Fifth International Congress for Applied Mechanics, Harvard University and MIT, 1938 (Wiley, New York, 1939)

[6] J. Nikuradse, Gesetzmässigkeiten der turbulenten Stromung in glatten Rohren (1932) [Laws of turbulent flow in smooth pipes, Report No. NASA TT F-10359, 1966].

[7] H. Schlichting, Boundary Layer Theory, fourth ed., McGraw-Hill, 1960 translated by J. Kestin.

[8] L.D. Landau and E. M. Lifshitz, Fluid Mechanics (2nd ed.) (Butterworth-Heinemann, 1987).

[9] B. H. Sun, Thirty years of turbulence study in China. Applied Mathematics and Mechanics, 40(2):193-214(2019).

[10] Z.S. SHE and Z.X ZHANG, Universal hierarchical symmetry for turbulence and general multi-scale fluctuation systems. Acta Mechanica Sin. 25 (3), 279 - 294(2009).

[11] SHE, Z. S., CHEN, X., WU, Y. and HUSSAIN, F. New perspective in statistical modeling of wall-bounded turbulence. Acta Mechanica Sin. 26 (6), 847 - 861(2010).

[12] SHE, Z. S., WU, Y., CHEN, X. and HUSSAIN, F. A multi-state description of roughness effects in turbulent pipe flow. New J. Phys. 14 (9), 093054(2012).

[13] CHEN, X. and SHE, Z. S. Analytic prediction for planar turbulent boundary layers. Science China Physics, Mech. Astron. 59 (11), 114711 (2016).

[14] Z.S. She, X. Chen and F. Hussain, Quantifying wall turbulence via a symmetry approach: a Lie group theory. J. Fluid Mech. 827, 322 - 356 (2017).

[15] X. Chen, F. Hussain and Z.S. She, Quantifying wal1 turbulence via a symmetry approach. Part 2. Reynolds stresses. J. Fluid Mech. 850, $401-438$ (2018).

[16] R. Baidya, J. Philip, N. Hutchins, J. P. Monty, and I. Marusic, Distance-from-the-wall scaling of turbulent motions in wall-bounded flows, Phy.Fluids 29, 020712 (2017)

[17] P. Luchini, Universality of the turbulent velocity profile, Phys.Rev.Lett. 118,224501(2017). 
[18] P. Schlatta, R. Örlü, Q. Li, G. Brethouwer, J.H.M. Fransson, A.V. Johnsson, P.H. Alfredsson and D.S. Henningson, Turbulent boundary layers up to $R e_{\theta}=2500$ studied through simulation and experiment. Phys. Fluids 21, 051702 (2019).

[19] P. R. Spalart, Direct simulation of a turbulent boundary layer up to $R_{\theta}=1410$, J. Fluid Mech. 187, 61 (1988).

[20] G. G. Stokes, On the effect of the internal friction of fluids on the motion of pendulums, Trans. Cambridge Philos. Soc, Vol. 9, 1851.

[21] E.R. van Driest, On turbulent flow near a wall. J. Aeronaut. Sci. (Institute of the Aeronautical Sciences) 23 (11), 1007 - 1011 (1956).

[22] J. Laufer, The structure of turbulence in fully developed pipe flow, NACA TN 2954, June, 1953.

[23] A. N. Kolmogorov, The local structure of turbulence in incompressible viscous fluid for very large Reynolds number. Dokl. Akad. Nauk SSSR, 30, 299-303 (1941a) (reprinted in Proc.R.Soc.Lond. A, 434,9-13, 1991)

[24] A. N. Kolmogorov, On degeneration (decay) of isotropic turbulence in an incompressible visous liquid. Dokl. Akad. Nauk SSSR, 31, 538-540 (1941b)
[25] A. N. Kolmogorov, Dissipation of energy in locally isotropic turbulence. Dokl.Akad. Nauk SSSR., 32, 1618 (1941c).(reprinted in Proc.R.Soc.Lond. A, 434,15-17, 1991).

[26] B. H. Sun, Scaling laws of compressible turbulence, Appl. Math. Mech. -Engl. Ed., 38(6), 765-778 (2017).

[27] W. T. Koiter, The stability of elastic equilibrium, Ph.D. thesis, Delft University of Technolog (1945).

[28] B.H. Sun, Buckling problems of sandwich shells, Delft University of Technology, Report LR-690, 1-99(1992).

[29] B.H. Sun, K.Y. Yeh and F.P.J. Rimrott, On the buckling of structures, Technische Mechanik, Band 12, Heft 2, 129140(1995).

[30] J. Arbocz, M Potier-Ferry and J. Singer, Buckling and Post-Buckling, Berlin Heidelberg, Springer (1987).

[31] Virot E, Kreilos T, Schneider T M, et al. Stability landscape of shell buckling, Phys.Rev.Lett, 119, 224101 (2017).

[32] A. Abramian, Virot E, et al. Nondestructive prediction of the buckling load of imperfect shells, Phys.Rev.Lett, 125,225504 (2020). 